\title{
Pavlovian appetitive conditioning under curare in rats*
}

\author{
EDWARD ZAMBLE $\dagger$ \\ Queen's University, Kingston, Ontario, Canada
}

\begin{abstract}
A CS was paired with rewarding intracranial stimulation while Ss were immobilized by curare. Tests of the activity response to the $\mathrm{CS}$ were carried out before and after conditioning. With either simple or discriminative conditioning, activity to the rewarded CS increased after training. These results are comparable to those in studies using food reward and support the hypothesis that a central motivational state is conditioned to stimuli preceding reward.
\end{abstract}

It has been repeatedly shown that a CS for food delivery develops the capacity to elicit increased skeletal activity. To the extent that it has been investigated, the assumption that the effect reflects a Pavlovian conditioning process seems entirely consistent with the data. For example, it is possible to demonstrate inhibition of delay of the learned activity response (Zamble, 1967).

These data have been used to support theories that postulate the control of instrumental responding by classically conditioned motivational elements (e.g., Sheffield, 1966; Mowrer, 1960). Generally, it is assumed that cues preceding appetitive rewards acquire motivational or emotional properties that allow them subsequently to control the pattern of instrumental responding. From this viewpoint, the "conditioned activity" phenomenon may be seen as a very useful index of a fundamental part of the reinforcement mechanism.

However, the evidence that the phenomenon observed represents a Pavlovian process is open to challenge. It may be argued that the application of Pavlovian procedures, which necessarily involves the use of noncontingent reinforcement, leads to the development of adventitious instrumental learning. It is the superstitiously learned behaviors that then produce the activity changes. Thus, the data might reflect only a minor case of instrumental learning and have little relevance to conditioned motivational responses.

It is most difficult to entirely counter this objection. However, it is possible to test specific formulations of the superstitious response argument and to limit the range of possible formulations.

In general, if conditioning operations carried out while a $S$ is paralyzed can be shown to affect responding, it is possible to dismiss mediation by simple sorts of gross motor responses. If increases in activity can be produced by conditioning under curare, explanations

*This research was carried out under a grant from the National Research Council of Canada. John Wiggin and Andrew Langford provided valuable assistance. The author would also like to thank Neal E. Miller and members of his laboratory for generous advice on techniques of curarization.

tRequests for reprints should be sent to Edward Zamble, Department of Psychology, Queen's University, Kingston, Ontario, Canada. involving adventitious reward will be limited to internal responses or central mechanisms (e.g., Black, Young, \& Batenchuk, 1970).

Thus, the purpose of the present research was to investigate the use of curare with Pavlovian conditioning operations using appetitive reinforcement. This work is similar to earlier studies involving conditioning under curare using aversive reinforcement (e.g., Solomon \& Turner, 1962).

\section{EXPERIMENT I}

\section{Method}

Subjects. Ss were six male Sprague-Dawley rats, approximately 1 year old and weighing $450-500 \mathrm{~g}$. They were maintained on food and water ad lib during the experiment. Data for two additional rats were excluded from analysis because they failed the test of intracranial self-stimulation, as described below.

Apparatus. Testing was carried out in boxes of aluminum and Plexiglas with grid floor $25 \times 22 \times 20 \mathrm{~cm}$ high. The box was dimly lit at all times, and white noise was present during all sessions in which data were taken. Activity was measured by means of a device described before (Zamble, 1967). Movements of the cage were transmitted mechanically to the movable coil of a 4-in. loudspeaker. Voltage from the speaker coil was amplified and used to trigger digital pulses. Every fourth pulse was selected by means of a binary counter and recorded on a printout counter. This recording system was especially sensitive to vigorous or sudden movements, but slow locomotion around the cage was also recorded.

Sensitivity of the activity recorder was continuously variable. Before and after every session, the sensitivity was calibrated by means of a metronome placed inside the cage and beating approximately 40 times per minute. The point of calibration was chosen to yield the highest possible level of recorded activity from movements around the cage without recording respiration or electrical noise. Changes of more than $10 \%$ from the preset level within a session were rare.

Testing for the effects of ICS was in a box of Plexiglas and stainless steel, $24 \times 22 \times 18 \mathrm{~cm}$ high. A nonfunctional foodcup and a space where a bar had been removed were on one side of the box. Testing was carried out in a quiet room with bright illumination.

During curarization the rat was placed on a small Plexiglas platform inside a sound-attenuating chamber. Respiration was given with a Physiograph Model V5KG respirator, and heart rate was monitored on a Beckman Type RB dynograph.

Stimuli presented in both testing and training situations were produced by Ameco tone generators. The same units were used for all sessions with a given $S$ to make stimuli as similar as 


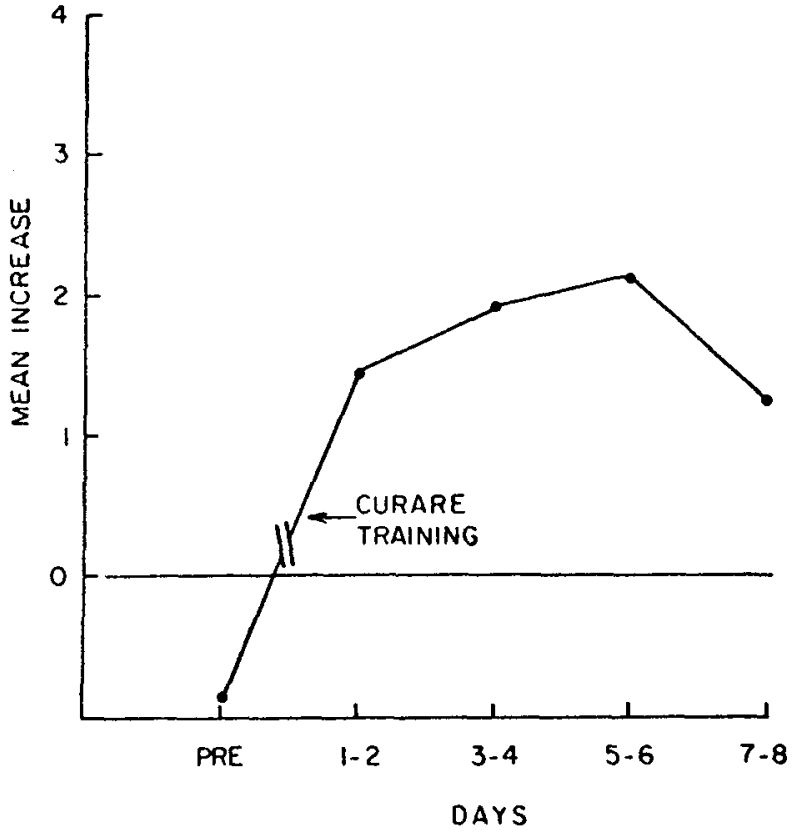

Fig. 1. Mean activity response to the CS in Experiment I before and after Pavlovian training.

possible in conditioning and test situations.

Procedure. Ss were first implanted bilaterally with monopolar electrodes aimed at the lateral hypothalamus, with coordinates $2 \mathrm{~mm}$ posterior to the bregma, $1.5 \mathrm{~mm}$ lateral to the midline, and $8.5 \mathrm{~mm}$ down from the top of the flat skull.

After 1 week to recover from the operation, each rat was given at least two 1-h sessions of habituation to the apparatus, during which total activity was measured. No stimuli were presented during these sessions.

Next, each rat was placed in the ICS test box and given a series of intracranial stimulations, $1 / 2-1 \mathrm{sec}$ long, from a $60-\mathrm{Hz}$ sine wave source. The stimulator was set initially at $20 \mu \mathrm{A}$ and the current level was slowly raised until $\mathrm{S}$ showed signs of activation (i.e., head movements, sniffing, and twitching of vibrissae) during stimulation. Both electrode placements were tested. The stimulator was then set at approximately $10 \mu \mathrm{A}$ above the activation threshold for the electrode that showed the better activation without visible motor effects; 60-75 stimulations, $1 / 2 \mathrm{sec}$ long, were delivered to this site, spaced randomly over a 15-min period.

Preliminary work had suggested that stimulation alone resulted in increased activity rates. To test for this effect, Ss were retested in the activity apparatus for six sessions after the preliminary ICS session to assess the effects on general activity levels. No large or consistent changes appeared, and the principal part of the study was begun.

Each rat was given two additional sessions in the activity apparatus. During each session there were eight presentations of an $800-\mathrm{Hz}$ tone, each $10 \mathrm{sec}$ long. The tones were spaced more or less equally throughout the session, except for the first $10 \mathrm{~min}$, during which no stimuli were presented. Activity preceding and during the tones was measured. These sessions constituted a pretest for the changes in activity produced by the tone before training.

Pavlovian training was then given to Ss immobilized by curare. An initial dose of .15-.20 cc of d-tubocurarine (Squibb, $3 \mathrm{mg} / \mathrm{cc}$ ) was injected intraperitoneally. As soon as the rat showed signs of difficulty in breathing, it was attached to the respirator by means of a mask made from a rubber balloon. Respiration was at the rate of 65 breaths/min, with a $1: 1 \mathrm{I} / \mathrm{E}$ ratio and a peak pressure of $18-20 \mathrm{~cm}$ of water. The rat was secured to the plastic platform. Electrodes for EKG recording were inserted subcutaneously, posterior to the shoulders and lateral to the heart; a ground electrode was clipped to the base of the tail. Last, ICS electrodes were attached.

At least $30 \mathrm{~min}$ after injection with curare and after heart rate had remained stable for a minimum of $10 \mathrm{~min}$, conditioning trials were begun. On each trial the $800-\mathrm{Hz}$ tone was presented alone for $10 \mathrm{sec}$, then remained on while a train of ICS was delivered. The ICS was at the level used earlier and consisted of three stimulations of $1 / 2 \mathrm{sec}$ separated by $50-60 \mathrm{msec}$. There were 80 conditioning trials. The intertrial varied between 30 and $60 \mathrm{sec}$, with an average of $45 \mathrm{sec}$.

In most cases, rats showed no visible signs of movement until 20-30 min after the end of conditioning. However, in a few cases conditioning was delayed and additional injections of .12-.15 cc of tubocurarine were administered.

After a day of recovery, Ss were tested again in the apparatus to determine the effects of conditioning. Postconditioning sessions were exactly like those in the pretest, i.e., there were eight presentations of the tone in each session. There were eight sessions in the posttest.

Finally, Ss were given a test for intracranial self-stimulation to determine the rewardingness of the ICS used in conditioning. Rats that could be trained either to press a bar or to nose a hole in the wall of the box were classed as self-stimulators. The minimum rate of responding required was $400 / \mathrm{h}$, although most Ss responded considerably faster. As stated before, two Ss failed this test and their data are not reported.

All Ss were sacrificed and gross histologies were performed to verify electrode location.

\section{Results}

There were no significant changes in overall activity rate following either preliminary ICS testing or curarization.

There were, however, changes in activity during the CS following training under curare. The "activity response" was defined as the difference between activity recorded during each $\mathrm{CS}$ presentation and that during the immediately preceding $10 \mathrm{sec}$. In order to reduce variance from individual rates of overall activity, each score was divided by the total daily activity for the respective day and rat. Responses for all trials on each test day were then averaged.

As can be seen in Fig. 1, the initial response to the tone was a small decrease in activity. This suppression effect approached statistical significance $(t=2.50$, $\mathrm{df}=5, \mathrm{p}<.06$, two-tailed). After curarization, however, the effect of the tone was to increase activity. For the average of the eight postcurare sessions, the activity response during the $\mathrm{CS}$ was significantly greater than zero $(t=2.64, d f=5, p<.05)$.

Since the effects of interest are the changes produced by training under curare, changes after conditioning were calculated by subtracting the mean activity responses on each postcurare session from pretraining values. Viewed in this way, the increase in activity during the CS after curarization was highly significant $(t=3.92, \mathrm{df}=5, \mathrm{p}<.01)$. The same statistic was also calculated for blocks of two test sessions and was significant to at least the .05 level on each of the four blocks.

It may be seen in Fig. 1 that the activity change during the CS reached its peak on Days 3-6 and then 
diminished on Days 7-8. The extinction effect on Day 8 was substantial and quite significant statistically (comparing Day 1 and Day 8, for example, $\mathrm{t}=4.64$, $\mathrm{df}=5, \mathrm{p}<.01)$.

\section{Discussion}

These results are quite consistent with a conditioned excitement hypothesis. There are consistent changes produced by conditioning during curarization that extinguish after several days of testing. Moreover, there were no other changes in activity as a result either of curarization or of ICS, so the effects were specific to the conditioning procedure.

However, the experimental design is not entirely convincing, since it does not rule out the possibility of sensitization. For this reason, a second study was carried out, using a stimulus not paired with ICS to control for possible sensitization or pseudoconditioning.

\section{EXPERIMENT II}

The second study was quite similar to the first, with the basic difference being the addition of discriminative Pavlovian training. Since the first experiment found no changes resulting from administration of ICS alone, the second study was simplified somewhat by omitting some of the preconditioning test procedures. In addition, activity during curarization was monitored to insure that there were no covert movements that could be adventitiously reinforced.

\section{Method}

Subjects. Ss were male Sprague-Dawley albino rats, approximately 1 year old. Ten Ss completed the experiment and are included in the analyses. Data for five others are not included because they failed to show satisfactory self-stimulation performance. An additional four Ss developed respiratory infections during pretraining, and they were discarded from the experiment before curarization.

Apparatus. The activity recording and ICS stimulation apparatus were the same as that used in Experiment $I$.

The curare apparatus was the same, with a single exception. In order to measure movement during curarization, an activity sensing device was placed below the platform on which $\mathrm{S}$ rested. Movements of the rat caused inductive changes in a tuned circuit that were transformed into polygraph deflections. Even the smallest twitches visible to the eye were recordable, as were the rhythmic movements produced by artificial respiration. This arrangement served to supplement visual observations of $S$ and relieved $E$ of the need for continuous close visual monitoring to detect recovery from the curarized state. This measure was chosen in preference to EMG recording, since it is affected by any movements, not just those of particular muscle groups. It does not, however, measure isometric muscle activity.

One of the stimuli used in this experiment was the same $800-\mathrm{Hz}$ tone used previously. The other stimulus consisted of a clicking noise, at the rate of 100 clicks/min.

Procedure. Implantation of electrodes was carried out as in Experiment I, followed by a recovery period. Ss were then given two 1 -h sessions of initial habituation to the activity apparatus, during which only total activity scores for the session were recorded. Next, there were two sessions in which stimuli were presented to determine preconditioning responses to each stimulus. The click and the tone each occurred six times a session in a random sequence, with a minimum of $3 \mathrm{~min}$ between stimuli. Each stimulus was $10 \mathrm{sec}$ long.

Conditioning under curare was then carried out. Ss were given initial injections of .20-.25 cc of d-tubocurarine and respirated as before. At least $15 \mathrm{~min}$ after visible movements had ceased and after the heart rate had stabilized, conditioning was begun. One of the two stimuli was chosen as CS+ and was accompanied $10 \mathrm{sec}$ after its onset by a train of three bursts of ICS, each $1 / 2 \mathrm{sec}$ long and separated by $50-60 \mathrm{msec}$. The other stimulus was CSand was the same length as CS+ but was not accompanied by ICS. Of the 10 Ss whose data are reported, 6 had the tone as CSt and 4 had the click reinforced. There were 60 presentations of each stimulus presented in random sequence. The average intertrial interval was $2 \mathrm{~min}$, with the range of intervals $1-3 \mathrm{~min}$.

During curarization, both EKG and S's movement were monitored. Additional doses of curare $(.10-.15 \mathrm{cc})$ were given after any signs of recovery. However, additional doses were routinely given after half the training trials, and signs of recovery before the completion of training were rare.

After at least 1 day for recovery, Ss were tested again in the activity apparatus. The two stimuli were each presented six times, as in the pretest sessions. There were eight such test sessions.

Finally, Ss were tested for self-stimulation, as in Experiment I, and sacrificed for histological verification of electrode placements.

\section{Results}

Activity responses for each CS were obtained by subtracting counts during the $10 \mathrm{sec}$ preceding CS onset from those during the stimulus. These responses were then standardized and averaged, as in Experiment $I$.

Upon inspection, it appeared that responses to both stimuli were consistently greater than zero, in both the pretest and posttest sessions. Therefore, data were expressed as changes in the activity response after conditioning by subtracting values for each stimulus in the postcurare sessions from the pretest values.

As may be seen in Fig. 2, the graph for CSt on the first few test days is similar to the pattern of results in Experiment I, with a peak on Days 3-4. However, the effects were accompanied by a substantial amount of variance, and the changes do not reach statistical significance (for Days 1-4, .20 $<\mathrm{p}<.10$ ). Several Ss showed increasingly variable scores during the latter test days, resulting in the variable mean scores for Days 5-8 shown in the graph.

For CS- the effect decreases linearly over test days. However, the results are not significant even on the first block of trials $(.10<p<.05)$.

Since the results for either stimulus alone showed no significant changes, the two effects were combined. The changes from pretest to posttest for $\mathrm{CS}$ - were subtracted from those for CSt to form a measure of total change. The results for this measure, which may be seen in Fig. 2, are quite significantly greater than zero $(\mathrm{t}=3.04, \mathrm{df}=9, \mathrm{p}<.01)$. For Days $3-4$ the difference is smaller but still marginally significant $(\mathrm{t}=1.89, \mathrm{df}=9$, $p<.05$, one-tailed). Mean differences for Days $5-8$ do not approach significance, and there is a significant decline from the levels of the first four sessions: comparing Days 1.4 with Days 5-8, $t=2.75, \mathrm{df}=9$, $\mathrm{p}<.01$, one-tailed. 


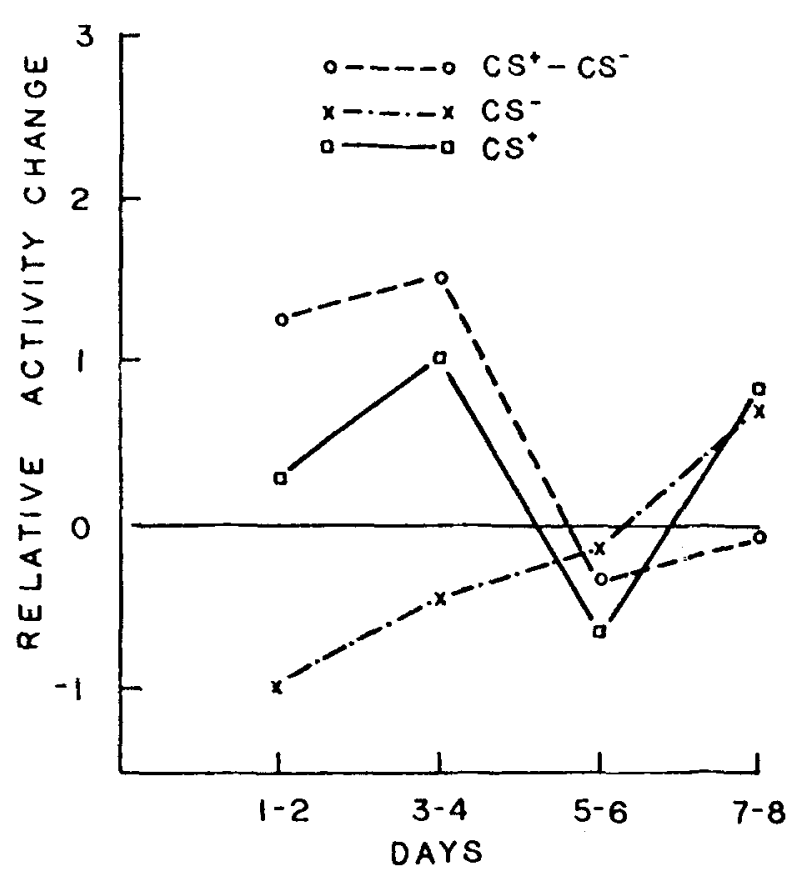

Fig. 2. Mean activity responses after training under curare for Ss in Experiment II.

\section{Discussion}

Since the results of neither CSt nor CS- are significant alone, it is impossible to determine for certain whether the overall effect is excitatory, inhibitory, or both. However, since the combined results are highly significant, it is certain that the conditioning procedure produced learned changes in the activity response.

It is not clear why the CS+ effect alone was not significant, as in Experiment I. The difference is perhaps a result of the number of trials used in discriminative conditioning, since there were fewer presentations of CS+ in Experiment II. However, examination of the data supports the assertion that the failure to reach significance was produced by a few Ss, which strongly increased the variance.

\section{GENERAL DISCUSSION}

The effects in the present experiments were fairly consistent and appeared in almost every S. The basic result is quite similar to those seen in previous studies using food as a reinforcer. Thus, it appears that a "conditioned activity" effect can be produced under conditions where instrumental mediation by gross motor behavior is unlikely.

It is, of course, still possible that invisibly small movements were adventitiously reinforced, but such responses would be unlikely to have produced the observed changes in gross motor activity. Similarly, tonic or isometric muscle activity may have been present, but these would not be likely to have had any significant effects on the activity measuring system employed in the test procedure.
Thus, an explanation based on the possibility of adventitious instrumental reinforcement of overt motor responses seems quite unlikely, and it seems simpler at this point to view the phenomenon as an index of a Pavlovian process. At the same time, the experiments reported here deal only with the most obvious sorts of responses. It is still possible that adventitious contingencies involving implicit or internal behaviors were present.

It is difficult to imagine how one might experimentally rule out such internal behavior in the present situation. While one might be able to demonstrate that any specified response was not responsible for the results reported here, it is not possible to show that other unspecified responses were not serving as instrumental mediators. Indeed, the problem only serves to highlight the general confusion in the current literature associated with the distinction between hypothesized Pavlovian and instrumental processes in learning. In any case, the experiments reported here are in no sense a final answer to the question, but they do help to narrow the range of defensible positions by ruling out the likelihood of simple skeletal mediation.

Whatever its ultimate classification in terms of a learning mechanism, the response acquired in these experiments occurs on a central level. Although the observed changes in behavior involved activity levels during the test sessions, the learning involved in producing these changes occurred during conditioning under curare. Thus, the activity changes could not have been themselves learned.

Instead, the activity changes reported here must reflect changes in some central excitatory state. It has been previously argued that general activity during a CS for reward is useful as an index of central excitement (e.g., Sheffield, 1966; Zamble, 1969). Regardless of one's view regarding the learning mechanism involved here, the present data support such an hypothesis.

This claim bears on a recent proposal by Trapold and Overmier (1972). They suggest that motivational or energistic considerations are perhaps unnecessary in considering the effects of Pavlovian operations on instrumental response processes. Trapold and Overmier propose that the results of transfer studies are most economically described within a notion of "transfer of control," without resort to motivational or emotional constructs.

However, if the activity changes reported here reflected changes in a central excitatory state, it would seem necessary to maintain at least some role for motivational concepts in this area. The use of activity as a dependent measure requires either an appeal to motivational considerations, e.g., activity is viewed as an index of motivational states, or a specification of how the independent variables directly affect the activity measure. With the use of curare, the latter is not easily accomplished for the present case. 
A final observation is prompted by the pattern of changes over days in the effect reported here. The results for CS+ in Experiment II and for the single CS in Experiment I are alike in showing the greatest change after several sessions, rather than at the beginning of testing. Although this pattern did not reach statistical reliability here, the result is very similar to a previous result (Zamble, 1969), and the repetition of the pattern leads one to believe that it may be a fairly general one in experiments of this sort. The reason for such a result is not apparent, but following the earlier report (Zamble, 1969), we may suggest that the central excitatory state conditioned by Pavlovian training does not immediately manifest itself as skeletal activity; rather, it must be "channeled" into responding and is seen as skeletal activation only after it occurs in the absence of any other available behavior, i.e., an instrumental response. If this idea can be substantiated, it might have some interesting implications for transfer studies.

\section{REFERENCES}

Black, A. H., Young, G. A., \& Batenchuk, C, Avoidance training of hippocampal theta waves in flaxedilized dogs and its relation to skeletal movement. Journal of Comparative \& Physiological Psychology, 1970, 70, 15-24.

Mowrer, O. H. Learning theory and behavior. New York: Wiley, 1960 .

Sheffield, F. D. New evidence on the drive induction theory of reinforcement. In $\mathrm{R}$. Haber (Ed.), Current research in motivation. New York: Holt, Rinehart, \& Winston, 1966. Pp. 111-122.

Solomon, R. L., \& Turner, L. H. Discriminative classical conditioning in dogs paralyzed by curare can later control discriminative avoidance responses in the normal state. Psychological Review, 1962,69, 202-218.

Trapold, M., \& Overmier, J. B. The second learning process in instrumental learning. In A. H. Black and W. R. Prokasy (Eds.), Classical conditioning II. New York: Appleton-Century-Crofts, 1972. Pp. 427-452.

Zamble, E. Classical conditioning of ex citement anticipatory to food reward. Journal of Comparative \& Physiological Psychology, 1967, 63, 526-529.

Zamble, E. Conditioned motivational patterns in instrumental responding of rats. Journal of Comparative \& Physiological Psychology, 1969, 69, 536-543.

(Received for publication A pril 16,1973: revision received November 12,1973 .) 